\title{
A New Target Radar Cross Section Based Passive Radar Surveillance Receiver Positioning Algorithm on Real Terrain Maps
}

\author{
Burak TUYSUZ \\ Dept. of Electrical and Electronics Engineering, Recep Tayyip Erdogan University, Rize, Turkey \\ burak.tuysuz@erdogan.edu.tr \\ Submitted January 18, 2018 / Accepted May 12, 2018
}

\begin{abstract}
Placing surveillance receivers on passive radar systems is a complex task because of the dependence on third party vendors. This paper presents an efficient and robust algorithm for positioning passive radar surveillance receivers for the intended coverage based on the available transmitters of opportunity in the area. First, by using the topology maps of the area of interest, depending on the expected targets and available transmitters, visibility maps of the terrain is generated with a line of sight algorithm. Then, in terms of the position and the orientation of the targets, the bistatic radar cross sections are determined. Finally, based on the bistatic radar cross sections of the targets, antenna characteristics, visibility analysis and received power levels, the optimum location for the surveillance receiver and the best transmitter of opportunity to be employed is decided. A practical scenario by using frequency modulation radio transmitters is realized in the manuscript for the rapid deployment of a mobile passive radar system. It is shown that on a terrain with multiple transmitters, depending on the characteristics of the transmitters and intended coverage, an optimum location for the surveillance receiver can be found for rapid deployment.
\end{abstract}

\section{Keywords}

Radar theory, passive radar, surveillance, radar receivers, radar cross section

\section{Introduction}

Ground-based radars are one of the most important components of the early-warning defense systems and many of the modern air and maritime surveillance systems suffer from blind zones created by the terrain features of the region. The potential coverage area gaps of the main surveillance system can be suppressed by the collaborative use of additional systems.

Passive radars can be utilized as auxiliary systems to cover the blind zones of the main surveillance systems while avoiding radio frequency (RF) emissions. Mainly, these systems exploit feasible transmitters (such as Digital Video Broadcast-Terrestrial (DVB-T), Global System for Mobile Communications (GSM) or Digital Audio Broadcasting (DAB)), called "illuminators of opportunity", in the region for their operation and make difference over the conventional radars. Especially in the last decade, investigations on the possible use of passive radar systems for surveillance purposes have received much attention from researchers because of the inherent advantages [1-3]. Also, the introduction of new wireless technologies and high power transmitters keep the interest in the field alive [4].

In a typical bistatic passive radar system, high signal to noise ratio (SNR) copy of the transmitted signal is collected by the reference receiver, and the surveillance receiver gathers echoes from the targets to perform detection and localization [5]. The reference receiver can be conveniently located on a place with a direct view to the transmitter. However, the direct signal, if received by the surveillance receivers, will mask weak echoes from the targets, introduce ambiguities and increase the dynamic range requirement. Thus, in passive radar systems, where the transmitter is not under the control of the radar designer, placing the surveillance receivers is a major challenge. In a well designed auxiliary passive radar system, the surveillance receiver should avoid direct signal reception and cover the gaps of the main surveillance system. In the literature, limited attempts have been made to assist the placement of surveillance receivers. Hoyuela et al. proposed a methodology for determining the possible receiver locations in which propagation losses are computed by the AREPS software [6]. However, the proposed algorithm assumed a constant radar cross section (RCS) for the targets and tested on simulated terrain data. It is also stated that for an efficient and accurate location determination algorithm, the RCS value should depend on the target geometry, target position, and locations of the transmitter and the receiver. On a study by Edrich et al. "a sophisticated mission planning tool" is developed for determining the best transmitter and receiver positions [7]. The tool is based on analytical models such as radar equation and has two modes that run in paralel to analyze an area of interest and particular reference 
trajectory for a given target. However limited information is given about the tool for further comparison. In another study by Tang et al., a randomized Voronoi algorithm is proposed for optimally placing a number of radar transmitters and receivers [8]. This study is focused on the optimization part without introducing a realistic model. These previous studies all concentrate on defense applications but the inherent reliability problems of the passive radars make them the perfect tool to probe the ionosphere for the long term and continuous observations [9]. An efficient algorithm which can guide any radar designer to determine the best surveillance receiver location while deploying the system to remote locations can increase the number of passive radar applications on ionospheric research and accelerate the process.

In this paper, based on the target RCSs and topology of the region, an efficient and robust surveillance receiver location determination algorithm is proposed. The proposed algorithm also specifies the most suitable transmitter in the region for the passive radar operation among multiple transmitters. First, the available illuminators to be exploited in the area are identified and the surrounding topology map is generated by using the real terrain data. Next, transmitters and sample targets, which are intended for detection or tracking, are placed on expected locations and altitude. Considering the location of the transmitters and the targets, a visibility map is generated by employing a line of sight algorithm. Then the targets are illuminated from the candidate locations of the visibility analysis and the relevant bistatic RCSs are predicted by using the physical optics (PO) approximation. Finally, an optimum location for the surveillance receiver is determined based on the target RCS, transmitter antenna properties, and the topology of the region.

The rest of the paper is organized as follows. In Sec. 2, a brief description of the bistatic radar equation is followed by the definitions of terrain generation, coordinate system and the RCS. Section 3 is dedicated to the algorithm description and a sample scenario to demonstrate the application of the algorithm is given. Finally, conclusions are drawn in Sec. 4.

\section{Preliminaries}

In this section, first, a brief review of the bistatic radars is given. It is followed by the description of the terrain generation algorithm, the coordinate system employed in the algorithm, and RCS computation by using the PO approximation.

\subsection{Bistatic Radar Equation}

The bistatic radar may be defined as a radar with spatially separated transmission and reception antennas [10]. Because of the non-dedicated transmitters employed in the system, passive radars fall under the category of bistatic radars [11]. A simple bistatic radar geometry is shown in Fig. 1. In the given figure, the transmitting antenna and the receiving antenna are separated by a distance $R_{\mathrm{B}}$, which is

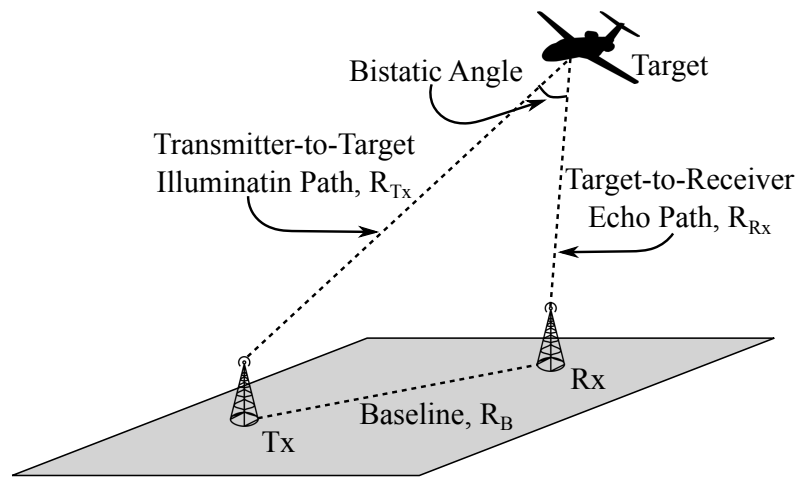

Fig. 1. Bistatic radar geometry.

called the baseline. The distances between the transmitterto-target and the target-to-receiver paths are called the illumination path, $R_{\mathrm{Tx}}$, and the echo path, $R_{\mathrm{Rx}}$, respectively. The vertex at the target, which is called the bistatic angle, is the unique difference between monostatic and bistatic radars and it is used to compute the bistatic RCS.

The received signal power, $P_{\mathrm{Rx}}$, in the given radar geometry can be represented as;

$$
P_{\mathrm{Rx}}=\frac{P_{\mathrm{Tx}} G_{\mathrm{Tx}} G_{\mathrm{Rx}} \lambda^{2} \sigma_{\mathrm{B}}}{(4 \pi)^{3} R_{\mathrm{Tx}}{ }^{2} R_{\mathrm{Rx}}{ }^{2} L} .
$$

In the above expression, $P_{\mathrm{Tx}}$ denotes the transmitter power. The antennas of the system are identified by the transmitter antenna gain, $G_{\mathrm{Tx}}$, and the receiver antenna power gain, $G_{\mathrm{Rx}}$. The radar wavelength is shown as $\lambda$ and $\sigma_{\mathrm{B}}$ represents the bistatic RCS of the target. In the equation, $L$ denotes the general loss factor for atmospheric, propagation and processing losses.

The signal to noise ratio (SNR) is the ratio of the received power, $P_{\mathrm{Rx}}$, to the input noise power of the receiver, $N$, which is defined as follows:

$$
N=k T_{\mathrm{e}} B
$$

where $k$ is the Boltzmann's constant, $T_{\mathrm{e}}$ is the effective system noise temperature in Kelvin and $B$ is the receiver noise bandwidth.

\subsection{Terrain Generation and Coordinate System}

Employment of real terrain maps on the placement determination of the surveillance receivers provide opportunities to accurately test and escalate the usability of the algorithm. In this work, the Shuttle Radar Topography Mission (SRTM) elevation data of the National Aeronautics and Space Administration (NASA) is used to generate the terrain [12]. The publicly available SRTM 3 version of this data set has 3 arc-seconds resolution and the elevations are calculated relative to the WGS84 ellipsoid, which is the default datum used for the Global Positioning System (GPS). 


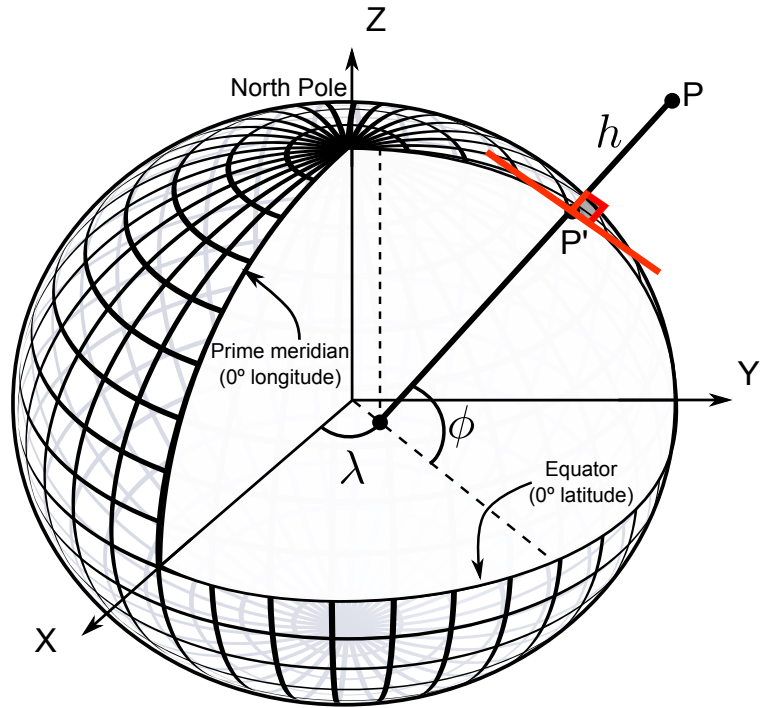

Fig. 2. Geodetic coordinate system.

In this study, the WGS84 geodetic coordinate system is used to pinpoint the transmitters, radar receivers and the targets on the terrain. The schematic of the coordinate system and its parameters are shown in Fig. 2. In the figure, $X, Y$ and $\mathrm{Z}$ axis describes the Earth Centric Earth Fixed (ECEF) coordinates. At an arbitrary point $\mathrm{P}$, the distance from the normal line at the point on the surface of the Earth is defined as height, $h$, and the intersection point is shown as $\mathrm{P}^{\prime}$ on the figure. The term $\phi$, geodetic latitude, is the angle between the equatorial plane and the perpendicular line that intersects P'. The term $\lambda$, geodetic longitude, is the angle in the equatorial plane between prime meridian and projection of the $\mathrm{P}^{\prime}$ on the $\mathrm{X}-\mathrm{Y}$ plane.

\subsection{Radar Cross Section}

The RCS is an important characterization parameter for the electromagnetic scattering properties of the objects which define the amount of energy reflected towards the radar receiver. It depends on several factors such as object orientation, size, geometry, material composition, radar frequency and polarization of the electromagnetic signal. Additionally, in a bistatic radar system, the RCS of an object depends on the transmitter and the receiver coordinates in order to determine the bistatic angle [13].

Computation of the RCS, except for some very simple surfaces, is complicated and can only be numerically approximated by computationally expensive methods [14]. Among these methods, the PO is one of the commonly used high-frequency asymptotic RCS calculation technique and it is applicable when the characteristic length of the body is much bigger than the wavelength of the incident wave $(L \gg 10 \lambda)$ [15]. The method is based on the integration of surface currents where each part of the target scatters energy independently from all other parts. On every illuminated surface point on a conducting plane, impinging plane wave nullifies the tangential component of the surface electric field and doubles the tangential component of the magnetic field.
Accordingly, the surface current on the shadowed portions is abruptly set to zero. Hence, PO currents of the conducting body, $\overrightarrow{\boldsymbol{J}}_{\mathrm{s}}$, can be found as;

$$
\overrightarrow{\boldsymbol{J}}_{\mathrm{s}}= \begin{cases}2 \hat{\boldsymbol{n}} \times \overrightarrow{\boldsymbol{H}}^{\mathrm{i}} & , \text { Illuminated region } \\ 0 & , \text { Shadow region }\end{cases}
$$

where $\overrightarrow{\boldsymbol{H}}^{\mathrm{i}}$ is the incident magnetic field intensity and $\hat{\boldsymbol{n}}$ is normal to the surface. The surface current from portions is then integrated to compute the scattered field far from the target [16].

In this study, the POFACETS tool, which utilizes the PO method, is employed for far field bistatic RCS approximation of the targets [17]. First, the surface currents on the triangular facets which comprise the building blocks of the target model are computed. Then, the bistatic RCS of the target is approximated by the radiation integrals.

\section{Proposed Algorithm}

The proposed algorithm for the determination of the best receiver location consists of five major building blocks as shown in Fig. 3. In the first block, the area of interes (AOI) is determined and then some model parameters are defined such as transmitter attributes in the AOI. In the terrain generation block, the elevation map of the AOI is constructed and the transmitters and the expected targets are located on the map. In the visibility analysis block, the possible receiver locations are restricted depending on the line of sight which in turn reduces the required computation time and the resources. In the SNR analysis block, the received power from transmitters on each of the predetermined candidate locations is calculated and the best location for the receiver placement is resolved and demonstrated on the map.

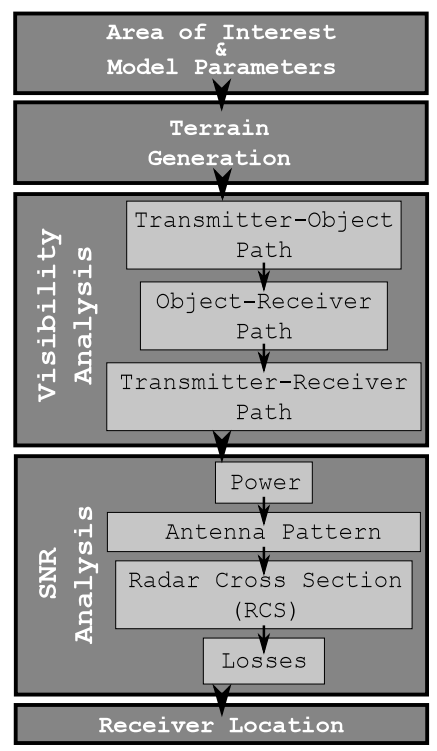

Fig. 3. Block diagram of the proposed RCS based passive radar surveillance receiver location determination algorithm. 


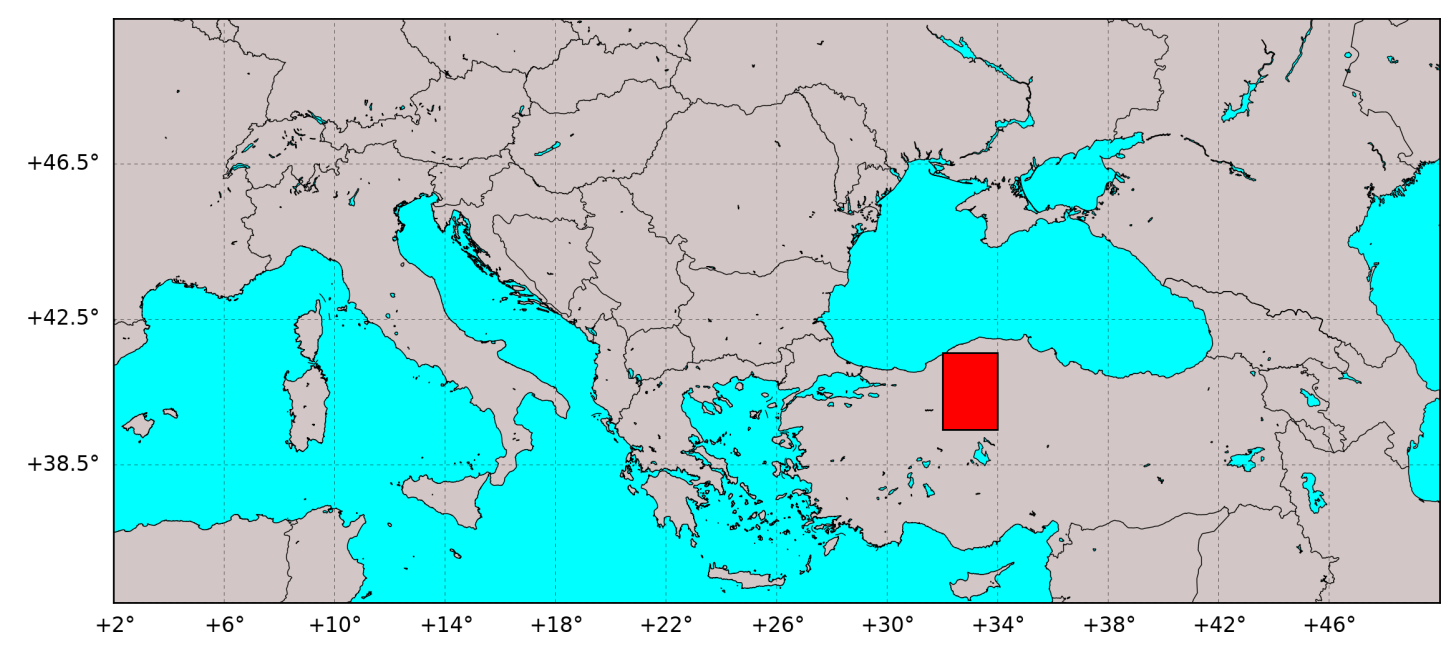

Fig. 4. Selected location of the area of interest rectangle, which spans about $42022.28 \mathrm{~km}^{2}$, is shown in red.

Each of the processing blocks of the proposed algorithm is reviewed separately for a sample scenario on following subsections. Diffusion, multi-path, and scattering along the terrain features have not been included in the algorithm. Also, focused beam emissions capable of reaching targets over-the-horizon, which is found in modern radar systems, is not explored in this study.

\subsection{Area of Interest and Model Parameters}

In the AOI identification block, first, the latitudelongitude pairs that define the AOI rectangle are determined. In this article, Central Anatolia Region of Turkey is chosen for the sample scenario in order to test the proposed algorithm. The borders of the AIO extends from $39.5^{\circ} \mathrm{N}$ to $41.6^{\circ} \mathrm{N}$ in latitude, and $32^{\circ} \mathrm{E}$ to $34.0^{\circ} \mathrm{E}$ in longitude, as shown in Fig. 4 . The area, which forms a $244.6 \mathrm{~km}$ by $171.8 \mathrm{~km}$ rectangle is shown in red and includes a mixture of mountains and low-lying ground that provides opportunities to gauge the performance of the algorithm. The AOI is sampled at every 3 arc-seconds, which is $1 / 1200^{\text {th }}$ of a degree, and a $1051-b y-1000$ matrix is constructed with latitude-longitude pairs of the corresponding locations.

In passive radar applications, frequency modulation (FM) radio transmitters are widely used because of the attractive ambiguity performance, relatively high transmitter power levels and being widespread [18]. For the sample scenario to demonstrate the performance of the proposed algorithm in this study, FM transmitters are preferred as the "transmitter of opportunity". Three FM radio transmitters are pre-selected for testing. The locations and the properties of these transmitters are given in Table 1. The effective radiated power of the FM transmitter 1, FM transmitter 2 and the FM transmitter 3 are specified as $100 \mathrm{~kW}, 98 \mathrm{~kW}$ and $99 \mathrm{~kW}$ at $98 \mathrm{MHz}, 102 \mathrm{MHz}$ and $90 \mathrm{MHz}$, respectively. The power levels of the transmitters are selected close to each other in order to emphasize the effects of the topology and RCS of the targets. All of the antennas are mounted at the same height above the ground and their effective bandwidth is specified as $50 \mathrm{kHz}$. The receiver antenna is a hypothetical linear phased array with $10 \mathrm{dBi}$ gain and placed $20 \mathrm{~m}$ above the ground. Additionally, the noise temperature is determined to be $300 \mathrm{~K}$. The system is assumed to operate with $1 \mathrm{~s}$ integration time which provides an additional $47 \mathrm{~dB}$ processing gain for $50 \mathrm{kHz}$ effective bandwidth.

Determination of the optimum surveillance receiver location is based on the given properties of the FM transmitters and the receiver. According to the radar equation in Equation $1, G_{\mathrm{Tx}}, R_{\mathrm{Tx}}, R_{\mathrm{Rx}}$ and $\sigma_{\mathrm{B}}$, are the target dependent variables and these parameters will be calculated within the algorithm.

\begin{tabular}{ll}
\hline Transmitter 3 (Tx3) Parameters & Value \\
\hline Effective Radiated Power (ERP) (Vertical Polarization) & $100 \mathrm{~kW}$ \\
Carrier Frequency & $98 \mathrm{MHz}$ \\
Transmitter Antenna Height Above Ground Level & $46 \mathrm{~m}$ \\
Effective Bandwidth & $50 \mathrm{kHz}$ \\
& \\
\hline Transmitter 1 (Tx1) Parameters & Value \\
\hline Effective Radiated Power (ERP) (Vertical Polarization) & $98 \mathrm{~kW}$ \\
Carrier Frequency & $102 \mathrm{MHz}$ \\
Transmitter Antenna Height Above Ground Level & $50 \mathrm{~m}$ \\
Effective Bandwidth & $50 \mathrm{kHz}$ \\
& \\
\hline Transmitter 2 (Tx2) Parameters & Value \\
\hline Effective Radiated Power (ERP) (Vertical Polarization) & $99 \mathrm{~kW}$ \\
Carrier Frequency & $90 \mathrm{MHz}$ \\
Transmitter Antenna Height Above Ground Level & $52 \mathrm{~m}$ \\
Effective Bandwidth & $50 \mathrm{kHz}$ \\
& \\
\hline Receiver Parameters & Value \\
\hline Integration Time & $1 \mathrm{~s}$ \\
Processing Gain & $\sim 47 \mathrm{~dB}$ \\
Noise Temperature & $300 \mathrm{~K}$ \\
Receiver Antena Height Above Ground Level & $20 \mathrm{~m}$ \\
Receive Antenna Gain (Gr) & $10 \mathrm{dBi}$ \\
\hline
\end{tabular}

Tab. 1. Simulation parameters of the transmitters and the receiver. 


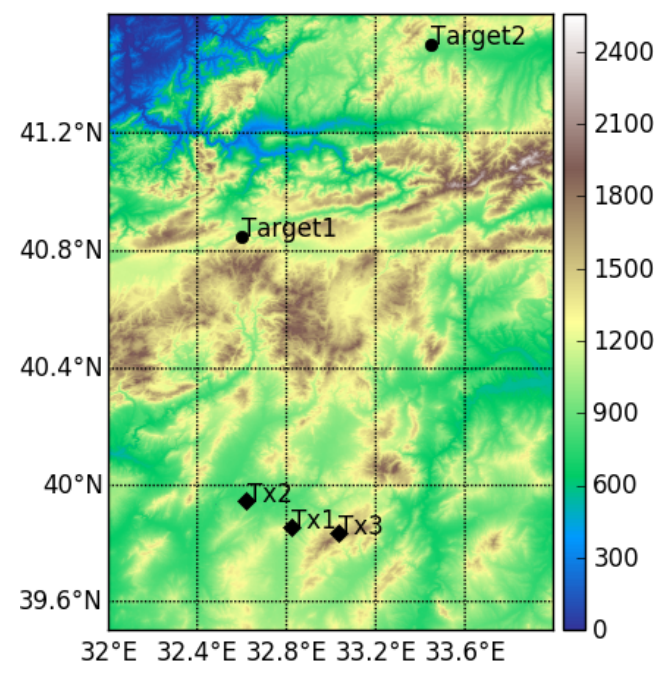

Fig. 5. The elevation map of the AOI. The publicly available STRM3 maps of NASA with $90 \mathrm{~m}$ resolution are used for the elevation information of the coordinates. The sea level is shown in dark blue and the higher elevations are gradually changed from blue to white. Three FM transmitters, Tx1, Tx2, Tx 3 and the targets Target 1 and Target2 are also placed on the map.

\subsection{Terrain Generation}

The elevation map is formed by importing the elevation information of the corresponding AOI locations from the SRTM3 model. The generated elevation map is shown in Fig. 5. In the map, gradual colors from dark blue to white are used to represent heights. The dark blue color represents the sea level. Gradually, the blue color is followed by the green and the yellow, which represent the heights from 100 meters to nearly 1300 meters. The heights above 1400 meters are represented in shades of the brown and the heights above 2400 meters are represented in white.

Additionally, the FM transmitters and the targets are placed on the elevation map in Fig. 5. In the figure, the FM transmitter 1 is labeled as Tx1 and located at $39.8561^{\circ} \mathrm{N}$, $32.8222^{\circ} \mathrm{E}$, the FM transmitter 2 is labeled as Tx 2 and located at $39.9463^{\circ} \mathrm{N}, 32.6225^{\circ} \mathrm{E}$ and the $\mathrm{FM}$ transmitter 3 is labeled as $\mathrm{Tx} 3$ and located at $39.8362^{\circ} \mathrm{N}, 33.0321^{\circ} \mathrm{E}$. The targets are arbitrarily placed at $40.85^{\circ} \mathrm{N}, 32.60^{\circ} \mathrm{E}$ at $6.0 \mathrm{~km}$ of altitude and $41.5^{\circ} \mathrm{N}, 33.45^{\circ} \mathrm{E}$ at $7.4 \mathrm{~km}$ above the mean sea level and marked as Target 1 and Target 2, respectively.

\subsection{Visibility Analysis}

In general, for the ideal operation of the bi-static radar systems, the line of sight must remain clear both in the illumination and in the echo paths. However, a direct line of sight in the baseline path will result in direct signal reception. Thus, additional signal processing techniques or antenna designs will be required for the cancellation of the direct path interference (DPI) [19-21]. In the algorithm, based on the topography of the AOI, the visibility analysis ensures that the unobstructed line of sight in the illumination and echo paths exist and the direct signal reception at the surveillance receiver is blocked on the candidate receiver locations.

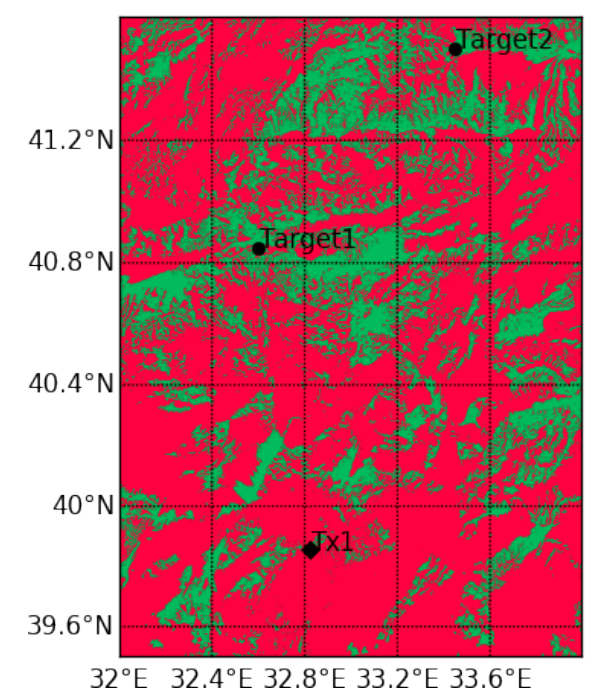

Fig. 6. Generated visibility map of the AOI for FM transmitter 1. The map is produced depending on the locations of the two targets, Target 1 and Target 2 and the terrain. Green color represents the available locations for the surveillance receiver placement and the red color represents the unavailable locations.

The applied line of sight algorithm checks whether the line connecting the observer and the target is above the terrain. Based on step size, 3 arc-seconds for this sample scenario, the connecting line is iterated and on each iteration the elevation is checked to see if there is any obstruction along the path. If there is any physical obstruction along the path, it is decided that the target is not visible from the observer on the real terrain.

Every location on the AOI is subjected to the visibility testing for each target. For a candidate receiver location, first, illumination and echo paths are checked for visibility. If there is no line of sight, further analysis is omitted and the location is marked as unsuitable. However, if the line of sight is achieved at the prospective receiver location, baseline path is analyzed for any obstruction and the location is marked as suitable on the visibility map if there is no direct line of sight. The generated visibility map for the FM transmitter 1 of the sample scenario is shown in Fig. 6. The green color represents the locations that have a direct line of sight to both targets and are not visible from the transmitter location. The red color indicates unsuitable locations for the receiver placement and these locations will be discarded in further steps of the receiver location determination analysis. The same line of sight algorithm is applied for each transmitter to produce individual visibility maps.

\subsection{Signal-to-Noise Ratio Analysis}

The SNR analysis block, based on the transmitter characteristics, target RCSs, and losses, calculates the SNR of each prospective receiver location. In the SNR analysis, target dependent variables $G_{\mathrm{Tx}}, R_{\mathrm{Tx}}, R_{\mathrm{Rx}}$ and $\sigma_{\mathrm{B}}$ of (1) are calculated individually for each candidate receiver location in order to estimate the received power and the SNR from each transmitter. 
The radar operator, instead of using the entire map, can usually limit the receiver location to a boundary based on the priorities and characteristics of the prospective system. These limits can be defined prior to the visibility analysis or the operator can make the decision depending on the visibility map. In the sample scenario, it is assumed that the candidate receiver location is defined after the visibility analysis and it is expected to be within $40.0^{\circ} \mathrm{N}$ to $40.4^{\circ} \mathrm{N}$ in latitude, and $32.0^{\circ} \mathrm{E}$ to $32.4^{\circ} \mathrm{E}$ in longitude.

First, for each candidate receiver location within the defined boundary of the visibility map, the length of the illumination, $R_{\mathrm{Tx}}$, and echo paths, $R_{\mathrm{Rx}}$, are calculated. The distance of the arbitrarily placed targets, Target 1 and Target 2 , to the FM transmitter $1, R_{\mathrm{Tx}}$, are found as $112 \mathrm{~km}$ and $190 \mathrm{~km}$ respectively. The distance of the targets to the candidate receiver location, $R_{\mathrm{Rx}}$, are dynamically calculated for each candidate location in the algorithm.

Secondly, the bi-static RCSs of the targets are approximated. For the calculation of the target RCSs, target models and the incidence angles are required. For the sample scenario, a hypothetical twin-engine airliner is modeled to define both the Target 1 and the Target 2, which is shown in Fig. 7. The given model is a triangular surface meshed in STL file format for RCS analysis and includes 10247 vertices and 20482 faces. In the given figure, the length of the model airliner is $30 \mathrm{~m}$ and the wingspan is $25 \mathrm{~m}$.

The nose on incidence angles on targets are computed by transforming the WGS 84 coordinates of the FM transmitter 1 and target locations to ECEF coordinate system. Then, the elevation and azimuth at the target can be computed by trigonometric identities. According to the target and transmitter locations, the nose-on incidence angle on Target 1 is $+90.800^{\circ} \theta,+16.550^{\circ} \phi$ and the nose-on incidence angle on Target 2 is $+90.350^{\circ} \theta,-26.446^{\circ} \phi$, in spherical coordinate system.

Conforming to the incidence angle and geometry, where forward-scatter is not explored, back-scatter can only be occurred within $\left[+90^{\circ},+180^{\circ}\right] \theta$ and $\left[-90^{\circ},+90^{\circ}\right] \phi$. Accordingly, the RCS of the targets can be predicted for the defined bistatic angle range at the operating frequency. The bistatic RCS of both targets at specific incidence angles are approximated with $1^{\circ}$ resolution by using the POFACETS program. The approximated bistatic $90^{\circ} \theta$-cut and $0^{\circ} \phi$-cut of the target RCSs are shown in Fig. 8. In the figures, each of the subfigures shows the incidence angle in elevation and azimuth planes. For computational convenience, both the incident and the scattered fields are simulated vertically polarized and the RCS approximations are stored in separate matrices for each target for the further computations.

Thirdly, the antenna pattern of the FM transmitter 1 is defined in the algorithm to be used in the calculations. In the sample scenario the antenna pattern of a dipole is used for all transmitters and according to the illumination angle relevant attenuation values are passed to the algorithm. The received power in all of the candidate receiver locations is used to construct the power map. For each of the candidate

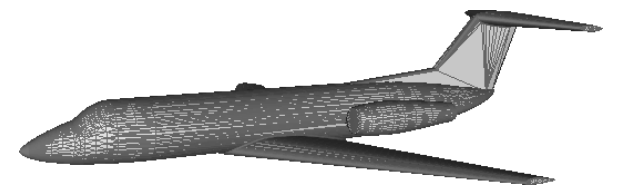

Fig. 7. The 3D discrete model of a small twin-engine aircraft in STL (STereoLithography) file format. The length of the model airliner is $30 \mathrm{~m}$ and the wingspan is $25 \mathrm{~m}$.

location, the incidence angle to the target and the reflection angle from the target to the receiver location is determined and the RCS, receiver antenna attenuation, $R_{\mathrm{Tx}}, R_{\mathrm{Rx}}$ values are combined with the simulation parameters in Tab. 1 to compute the SNR value of the candidate receiver locations for FM transmitter 1. Same steps are repeated for each transmitter to compute the individual SNR maps.

\section{Receiver Location}

The receiver location block determines the most suitable transmitter and the best possible receiver location with the calculated SNR values. There are four scenarios for the receiver location determination which are, single transmitter - single target, single transmitter - multiple target, multiple transmitter - single target and multiple transmitter - multiple target. For the single tranmitter-single target case, only one SNR value is possible for prospective receiver locations. Thus, the optimum receiver location is determined with the highest SNR. In case of single transmitter - multiple target situations, depending on the number of targets, multiple SNR values will be computed for each prospective location. The proposed algorithm stores the lowest SNR value computed for every location to account for the worst case scenario and the optimum receiver location is chosen depending on the highest SNR value. Additionally, for multiple transmitter cases, which are multiple transmitter - single target and multiple transmitter - multiple target, an SNR map is created for each transmitter individually and the highest SNR value on each SNR map is compared for selecting the best transmitter for the best receiver point. In this case, the receiver location will change for different transmittters. However, the optimum transmitter and receiver location can be found by checking the created SNR maps with highest SNR.

In the sample scenario, there are three transmitters and two targets. An SNR map is produced for each of the transmitters and the highest SNR values on the AOIs are noted. The transmitter with the highest SNR on the AOI is chosen as suitable and the location with the highest SNR on that map is decided for the receiver placement. The generated power map for the FM transmitter 1 is shown in Fig. 9. In the figure, the received power on the AOI, which is the intended area for deployment, changes from $42.4 \mathrm{~dB}$ to $57 \mathrm{~dB}$. The best receiver location is determined as $40.2787^{\circ} \mathrm{N}$ latitude and $33.4872^{\circ} \mathrm{E}$ longitude. On the simulation, the maximum power levels from FM transmitter 2 and FM transmitter 3 are recorded as $54 \mathrm{~dB}$ and $56 \mathrm{~dB}$, respectively. Thus, the FM transmitter 1 is selected as the most suitable transmitter and the receiver location is determined for this transmitter. 

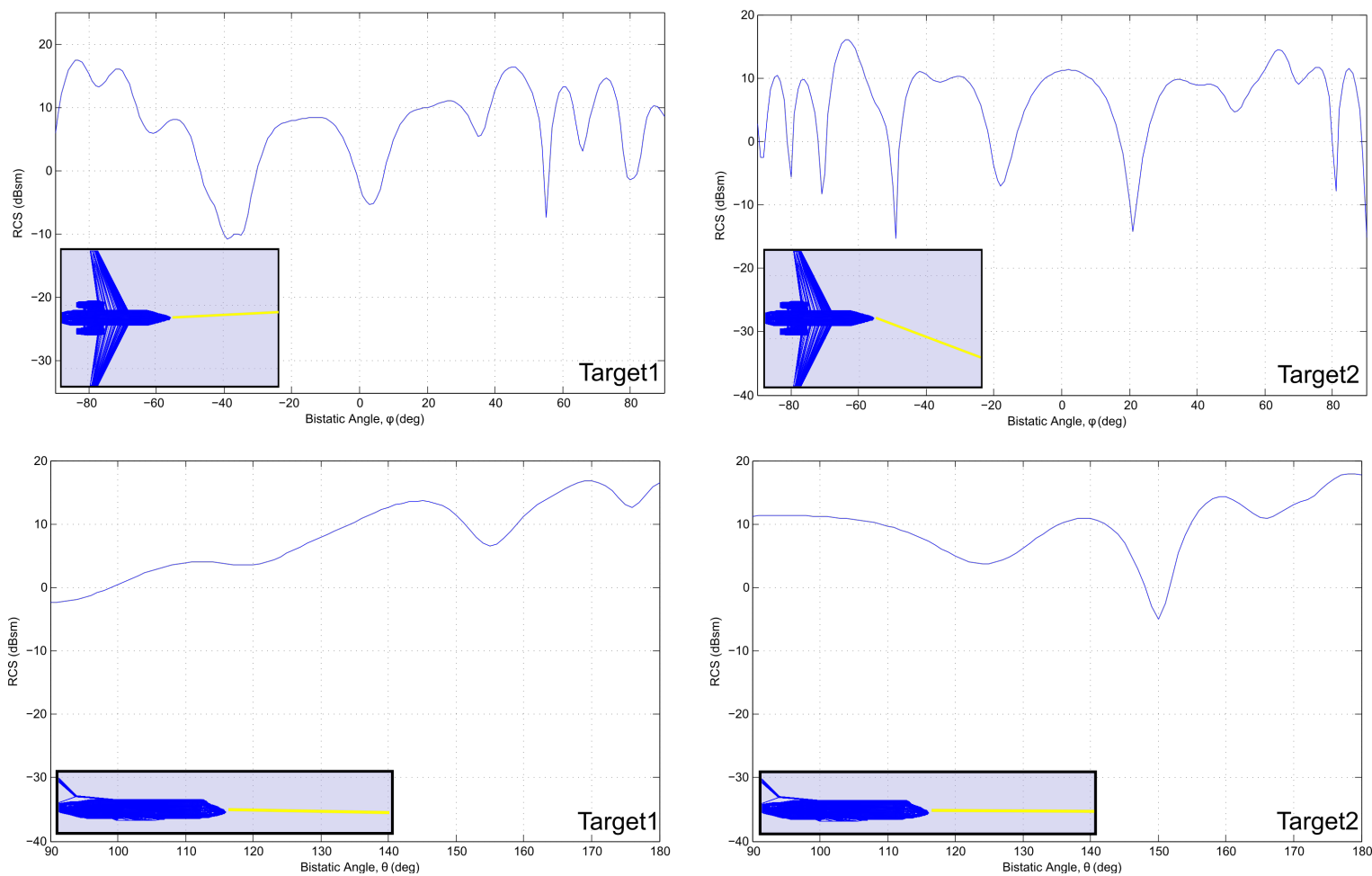

Fig. 8. The $90^{\circ} \theta$-cuts and $0^{\circ} \phi$-cuts of the target airliner RCSs. The incidence angle is shown with the yellow line in sub-figures and the RCS, which is computationally determined with POFACETS, is drawn in blue color. The incidence angle on Target 1 , which is shown on the left two sub-figures, is $+90.800^{\circ} \theta,+16.550^{\circ} \phi$. The incidence angle on Target 2, which is shown on the right two sub-figures, is $+90.350^{\circ} \theta,-26.446^{\circ} \phi$. The figures on top, shows the $90^{\circ} \theta$-cuts and the below two figures demonstrate the $0^{\circ} \phi$-cuts of the target RCSs according to the bistatic angle.

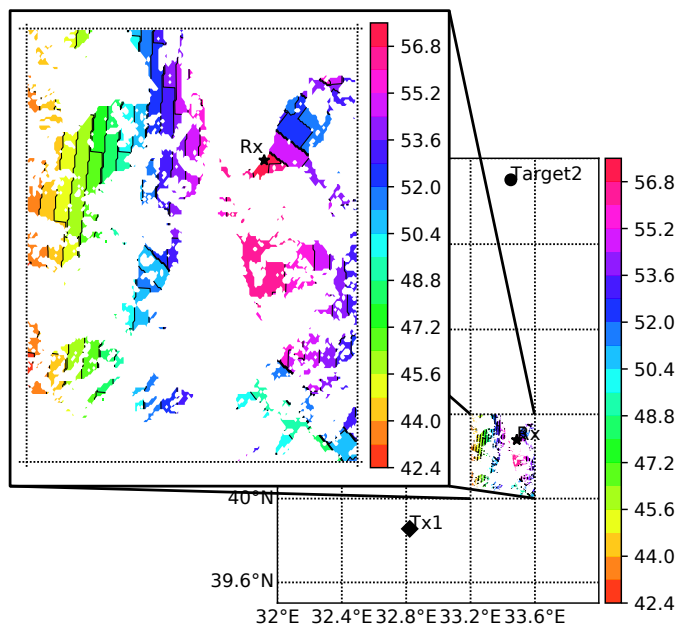

Fig. 9. The power map shows the received power from FM transmitter 1 depending on the terrain, target location, and the RCS of the targets.

\section{Conclusion}

Passive radar systems are becoming very popular and finding many application areas from atmospheric science to indoor security. However, the dependence on the third party transmitters makes passive radars unreliable to be used as the main surveillance systems. This papers mainly focuses on the determination of the best surveillance receiver location for passive radars. However, it particularly focuses on the possible use of passive radars as auxiliary systems to fill the coverage gaps or enhance the coverage of the main surveillance systems.

The proposed algorithm outputs the expected SNR values for multiple transmitters on the terrain and resolves the best location with highest SNR for the passive radar receiver site. The algorithm also determines the best transmitter in the area to be employed. It is shown that, depending on the multiple targets, transmitter properties, and terrain features, a very accurate location determination was made. For the selected scenario presented on this paper, the best location for the surveillance receiver of the passive radar is determined as $40.2787^{\circ} \mathrm{N}$ in latitude and $33.4872^{\circ} \mathrm{E}$ in longitude with $57 \mathrm{~dB}$ power level. This power level was achieved by employing the FM transmitter 1. The maximum power levels for FM transmitter 2 and FM transmitter 3 were recorded as $54 \mathrm{~dB}$ and $56 \mathrm{~dB}$, respectively.

The main benefits of the algorithm are the target RCS dependent operation and the use of real terrain maps. Use of high-resolution SRTM3 maps enhances the usability of the algorithm on real life situations. In addition, the target dependent operation increases the reliability of the output of the algorithm by including RCS as a variable. Another very important benefit of the algorithm is the multiple transmitter support. Among many transmitters in the area, the algorithm can be used to select the best possible transmitter as the transmitter of opportunity. The algorithm can also be 
used with different kind of emitters such as GSM and DVB-T. The antenna type of the transmitter can be selected from the company released 3D pattern files which greatly enhances the planning accuracy as it simulates true radiation capability. The presented algorithm also gives to the radar controller an idea about the expected power levels from targets.

Overall, readily available transmitters wait to be exploited for passive radar applications and one of the most important benefits of passive radars is the ability to use commercial frequency bands. Increasing passive radar application areas, especially in surveillance systems, will lower the operational and installation costs. Inherent reliability problems of the passive radars limit the use on critical operations. However, as auxiliary systems, passive radars can be supplementary to the main surveillance systems.

\section{Acknowledgments}

This work is supported by the Scientific and Technological Research Council of Turkey (TÜBİTAK) under grant $117 \mathrm{E} 008$.

\section{References}

[1] CAPRIA, A., CONTI, M., PETRI, D., et al. Ship detection with DVB-T software defined passive radar. In Proceedings of the IEEE GOLD Remote Sensing Conference, 2010, p. 3-5.

[2] SUN, H., TAN, D. K., LU, Y., LESTURGIE, M. Applications of passive surveillance radar system using cell phone base station illuminators. IEEE Aerospace and Electronic Systems Magazine, 2010, vol. 25, no. 3, p. 10-18. DOI: 10.1109/MAES.2010.5463951

[3] EDRICH, M., SCHROEDER, A. Multiband multistatic passive radar system for airspace surveillance: A step towards mature PCL implementations. In Proceedings of the International Conference on Radar. Adelaide (Australia), 2013, p. 218-223. DOI: 10.1109/RADAR.2013.6651988

[4] EVERS, A., JACKSON, J. A. Cross-ambiguity characterization of communication waveform features for passive radar. IEEE Transactions on Aerospace and Electronic Systems, 2015, vol. 51, no. 4, p. 3440-3455. DOI: 10.1109/TAES.2015.140622

[5] TUYSUZ, B., URBINA, J., LIND, F. D. Development of a passive VHF radar system using software-defined radio for equatorial plasma instability studies. Radio Science, 2013, vol. 48, no. 4, p. 416-426. DOI: $10.1002 /$ rds. 20047

[6] HOYUELA, C. M., TERZUOLI, A., WASKY, R. Determining possible receiver locations for passive radar. IEE ProceedingsRadar, Sonar and Navigation, 2005, vol. 152, no. 3, p. 206-214. DOI: $10.1049 /$ ip-rsn:20045023

[7] EDRICH, M., MEYER, F., SCHROEDER, A. Design and performance evaluation of a mature FM/DAB/DVB-t multi-illuminator passive radar system. IET Radar, Sonar \& Navigation, 2014, vol. 8, no. 2, p. 114-122. DOI: 10.1049/iet-rsn.2013.0162

[8] TANG, L., GONG, X., WU, J., ZHANG, J. Target detection in bistatic radar networks: Node placement and repeated security game. IEEE Transactions on Wireless Communications, 2013, vol. 12, no. 3, p. 1279-1289. DOI: 10.1109/TWC.2013.011713.120892
[9] TUYSUZ, B., URBINA, J., MATHEWS, J. Effects of the equatorial electrojet on FM-based passive radar systems. IEEE Transactions on Geoscience and Remote Sensing 2017, vol. 55, no. 7, p. 4082-4088. DOI: 10.1109/TGRS.2017.2687830

[10] WILLIS, N. J., GRIFFITHS, H. D. Advances in Bistatic Radar. SciTech Publishing, 2007. ISBN: 1891121480

[11] BEZOUSEK, P., SCHEJBAL, V. Bistatic and multistatic radar system. Radioengineering, 2008, vol. 17, no. 3, p. 53-59. ISSN: 1210-2512

[12] FARR, T. G., ROSEN, P. A., CARO, E., et al. The shuttle radar topography mission. Reviews of Geophysics, 2007, vol. 45, no. 2, p. 1-33. DOI: 10.1029/2005RG000183

[13] SKOLNIK, M. Radar Handbook. 3rd ed. New York (USA): McGrawHill Education, 2008. ISBN: 0071485473

[14] KNOTT, E. F., TUlEY, M. T., SHAEFFER, J. F. Radar Cross Section (Scitech Radar and Defense). SciTech Publishing, 2004. ISBN: 1891121251

[15] KOUYOUMJIAN, R. Asymptotic high-frequency methods. Proceedings of the IEEE, 1965, vol. 53, no. 8, p. 864-876. DOI: 10.1109/PROC.1965.4065

[16] BALANIS, C. A. Advanced Engineering Electromagnetics. Wiley, 1989. ISBN: 0471621943

[17] JENN, D. C., CHATZIGEORGIADIS, M. F. Asymptotic highfrequency methods. IEEE Antennas Propagation Magazine, vol. 46, no. 4 , p. $137-139$.

[18] BAKER, C. J., GRIFFITHS, H. D., PAPOUTSIS, I. Passive coherent location radar systems. Part 2: Waveform properties. IEE Proceedings - Radar, Sonar and Navigation, 2005, vol. 152, no. 3, p. 160-168. DOI: $10.1049 / \mathrm{ip}-\mathrm{rsn}: 20045083$

[19] COLONE, F., CARDINALI, R., LOMBARDO, P. Cancellation of clutter and multipath in passive radar using a sequential approach. In Proceedings of the IEEE Conference on Radar. Verona (USA), 2006, p. 393-399. DOI: 10.1109/RADAR.2006.1631830

[20] JIABING, Z., YI, H., LIANG, T. Adaptive beamforming passive radar based on FM radio transmitter. In Proceedings of the IET International Conference on Radar Systems. Edinburgh (UK), 2007, p. 13. DOI: $10.1049 / \mathrm{cp}: 20070488$

[21] TAO, R., WU, H., SHAN, T. Direct-path suppression by spatial filtering in digital television terrestrial broadcasting-based passive radar. IET Radar, Sonar \& Navigation, 2010, vol. 4, no. 6, p. 791-805. DOI: 10.1049/iet-rsn.2009.0138

\section{About the Authors...}

Burak TUYSUZ received his B.S. (with honors) and M.S. degree in Electrical and Electronics Engineering from Gaziantep University, Gaziantep, Turkey, in 2005 and 2007, respectively, and his Ph.D. degree in Electrical Engineering from Pennsylvania State University, University Park in 2013. His current research interests are in development of passive and active radar systems, software-defined radio, sensors, acquisition, compressive sensing, adaptive systems, radar signal processing, radio wave propagation, remote sensing, and radar studies of the atmosphere and ionosphere. 